\title{
The impact of delays in computed tomography of the brain on the accuracy of diagnosis and subsequent management in patients with minor stroke
}

\author{
J M Wardlaw, S L Keir, M S Dennis
}

J Neurol Neurosurg Psychiatry 2003;74:77-81

See end of article for authors' affiliations

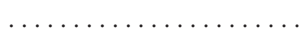

Correspondence to: Professor J Wardlaw, Department of Clinical Neurosciences, Western General Hospital, Crewe Road, Edinburgh EH4 2XU, UK:

jmw@skull.dcn.ed.ac.uk

Received 26 June 2002 In revised form 12 September 2002

Accepted 19 September 2002

\begin{abstract}
Objectives: To determine the proportion of haemorrhagic strokes misdiagnosed as infarcts on computed tomography $(C T)$ in patients with mild stroke, and the implications for health care.

Methods: Patients with mild stroke presenting as inpatients or outpatients four or more days after stroke to our stroke service (catchment population 500 000) were recruited prospectively. They underwent detailed clinical examination and brain imaging with CT and magnetic resonance imaging (MRI) on the day of presentation. CT and MR images were examined independently to identify infarct, primary intracerebral haemorrhage, haemorrhagic transformation, or non-vascular lesion.

Results: In 228 patients with mild stroke (median time from stroke to scan 20 days), primary intracerebral haemorrhage was identified by CT in two patients $(0.9 \% ; 95 \%$ confidence interval $(\mathrm{Cl}), 0.1 \%$ to $3.1 \%$ ) and $\mathrm{MRI}$ in eight (3.5\%; $1.5 \%$ to $6.8 \%)$. Haemorrhagic transformation was identified by $\mathrm{CT}$ in three patients $(1.3 \% ; 0.1 \%$ to $5.6 \%)$ and MRI in $15(6.6 \% ; 3.7 \%$ to $10.6 \%)$. The earliest time primary intracerebral haemorrhage was not identified on CT was 11 days.

Conclusions: CT failed to identify $75 \%$ of primary intracerebral haemorrhages, equivalent to 24 patients per $1000(95 \% \mathrm{Cl}, 14$ to 37) with mild strokes. To detect haemorrhages reliably, CT would need to have been performed within about eight days. Rapid access to neurovascular clinics with same day CT brain imaging is required to avoid inappropriate secondary prevention. Increased public awareness of the need to seek urgent medical attention after stroke should be encouraged. MRI should be considered in late presenting patients.
\end{abstract}

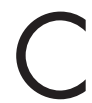
omputed tomography (CT) is the most common imaging method used to investigate stroke. It demonstrates haemorrhage accurately immediately after the stroke ${ }^{1}$ but as the haematoma is broken down, the characteristic hyperdensity on CT disappears, becoming indistinguishable from infarction. This has been reported to occur by 14 days for moderately sized lesions, ${ }^{2}$ and possibly sooner for smaller haemorrhages. ${ }^{3}$

Primary intracerebral haemorrhage is thought to cause more severe stroke symptoms than an infarct of the same size, ${ }^{45}$ and so primary intracerebral haemorrhage is considered to be a less frequent cause of mild than severe stroke. However, it is possible that this apparently low incidence is an artefact caused by the misclassification of mild stroke resulting from primary intracerebral haemorrhage as ischaemic stroke because of delay in CT scanning in epidemiological studies. ${ }^{6}$ The actual proportion of primary intracerebral haemorrhages among mild strokes is unknown. Thus there is no information on how many patients presenting around a week or more after the stroke, and with hypodense (apparently ischaemic) lesions on CT, actually had a primary intracerebral haemorrhage as the cause of their stroke.

Features of haemorrhage can remain visible on magnetic resonance imaging (MRI) for much longer. Signs of haemorrhage, as haemosiderin, can be detected for at least five years after (traumatic) intracerebral haemorrhage in most patients ${ }^{7}$ and probably are detectable indefinitely in the majority of patients if very blood sensitive MRI sequences (such as gradient echo) are used.

Patients with minor stroke symptoms may not be admitted to hospital, and may present days or weeks after stroke ${ }^{8}$ because the patient thinks their symptoms are unimportant. Other delays may occur while waiting for an appointment at hospital after seeing their family doctor, or waiting for the scan to be performed. However, for effective secondary prevention of stroke, distinction of ischaemic from haemorrhagic stroke is fundamental.

We investigated the potential scale of misdiagnosed primary intracerebral haemorrhage in patients presenting to a large teaching hospital neurovascular clinic. We aimed to determine what proportion of these patients had haemorrhage as the cause of their stroke and the latest time that CT could still reliably distinguish infarct from haemorrhage, and we assessed the acceptability of MRI as an alternative to CT.

\section{METHODS}

\section{Patient recruitment}

Between August 1998 and July 2000, consecutive patients presenting to our neurovascular service with minor stroke symptoms (first or recurrent stroke, but transient ischaemic attacks were excluded) were scanned on the day of presentation with CT and MRI. Most patients were outpatients.

All patients were assessed before scanning by an experienced stroke physician. They were classified according to the Oxfordshire community stroke project (OCSP) classification ${ }^{9}$ and the Canadian neurological score (CNS). ${ }^{10}$ The CNS is a neurological disability score ranging from 1.5 , indicating very severe stroke, to 10 , indicating no residual disability. We defined minor, or mild, stroke as partial anterior circulation syndrome or lacunar syndrome, or posterior circulation syndrome with no loss of consciousness or drowsiness and which did not cause any major disability. Medically unstable

Abbreviations: CNS, Canadian neurological score; OCSP, Oxfordshire community stroke project 
Table 1 Corresponding computed tomography findings in patients in whom some form of haemorrhage was identified on magnetic resonance imaging

\begin{tabular}{lll}
\hline & \multicolumn{2}{l}{ MRI findings } \\
\cline { 2 - 3 } CT findings & PICH & HTI \\
\hline PICH & 2 & 1 \\
HTI & 0 & 2 \\
Recent infarct & 5 & 10 \\
Old infarct & 1 & 1 \\
Asymptomatic (old) PICH & 0 & 0 \\
Other & 0 & 1 \\
Total & 8 & 15 \\
\hline
\end{tabular}

"Other" implies cortical atrophy or multiple periventricular lucencies only.

$\mathrm{CT}$, computed tomography; $\mathrm{HTI}$, haemorrhagic transformation of infarct; $\mathrm{MRI}$, magnetic resonance imaging; $\mathrm{PICH}$, primary intracerebral haemorrhage.

patients, and patients with absolute contraindications to MRI (pacemaker, aneurysm clip, known intraocular metal) were excluded at the time of assessment in the clinic, as they would not be able to undergo the MRI procedure.

\section{Imaging}

The order of scanning was determined by the availability of each scanner-that is, it was not random-but the scans were done one immediately after the other. For routine CT brain scanning a GE spiral scanner was used (total scan time from patient going into and coming out of the scanner about seven minutes). For MRI, one of two standard clinical scanners was used-up to October 1999 an Elscint Prestige 2T scanner; from January 2000, a GE 1.5 Signa Horizon LX scanner. Patients underwent routine structural MR imaging (Tl weighted sagittal, T2 weighted, proton density, FLAIR, and gradient echo axial sequences; imaging parameters are available on request from the authors), with the total scanning time in both MR scanners being approximately 25 minutes.

\section{Image analysis}

CT and MRI scans were printed onto $x$ ray film and read independently of each other, and blind to each result, on separate occasions at least four weeks apart, by one neuroradiologist who was given a brief clinical history. Scans were classified as showing: recent infarct; recent infarct with haemorrhagic transformation; recent primary intracerebral haemorrhage; old lesion probable infarct; old asymptomatic haemorrhage; another diagnosis (for example, tumour, multiple periventricular white matter lucencies, cortical atrophy, or no abnormality). "Recent" was taken to mean consistent with the time of onset of symptoms given in the brief clinical history. "Old" was taken to mean a lesion which appeared older than the clinical history suggested.

\section{Patient preference questionnaire}

Following completion of scanning, patients were asked about the acceptability of both scanning procedures, which scan they had undergone first, which they preferred, whether they would have either again if they had to, and for any comments about the procedures, including ways to make the scanning more pleasant.

\section{RESULTS}

Patient baseline characteristics, and acceptability of scanning

We recruited 232 patients, the majority being outpatients. MRI was not done in four patients $(1.7 \%$; three were claustrophobic and one was too large for the scanner). Therefore 228 patients had both CT and MRI on the same day. No images were considered uninterpretable.

The mean age of the patients was 67.5 years, median age 68 years (range 35 to 89 years). The median time from onset of stroke symptoms to presentation and scanning was 20 days (range 2 to 112). According to the OCSP classification, there were 95 partial anterior circulation strokes (41.7\%), 73 lacunar strokes $(32 \%)$, and 36 posterior circulation strokes ( $15.8 \%)$; in 24 cases $(10.5 \%)$ it was not possible to define a subtype. There were no total anterior circulation strokes. The median CNS was 9.5 (range 5.5 to 10 ), confirming that this population had mild strokes.

\section{Findings on CT and MRI}

MRI identified recent primary intracerebral haemorrhage compatible with the duration and location suggested by the symptoms and signs in eight patients $(3.5 \%$; $95 \%$ confidence interval (CI) $1.5 \%$ to $6.8 \%$ ). CT agreed in only two of these $(0.9 \% ; 0.1 \%$ to $3.1 \%$ ) (table 1$)$. Corresponding findings on CT when recent haemorrhage was not identified were: recent infarct (5) (fig 1) and old lesion probable infarct (1). The lesions diagnosed as ischaemic on CT were the same as those shown to be haemorrhagic on MRI. Thus, in patients with recent primary intracerebral haemorrhage identified on MRI, CT failed to make the diagnosis in six of eight $(75 \%)$.

MRI identified haemorrhagic transformation in 15 patients (6.6\%; $3.7 \%$ to $10.6 \%$ ). The extent of haemorrhagic changes seen varied from minor petechial spots to more extensive
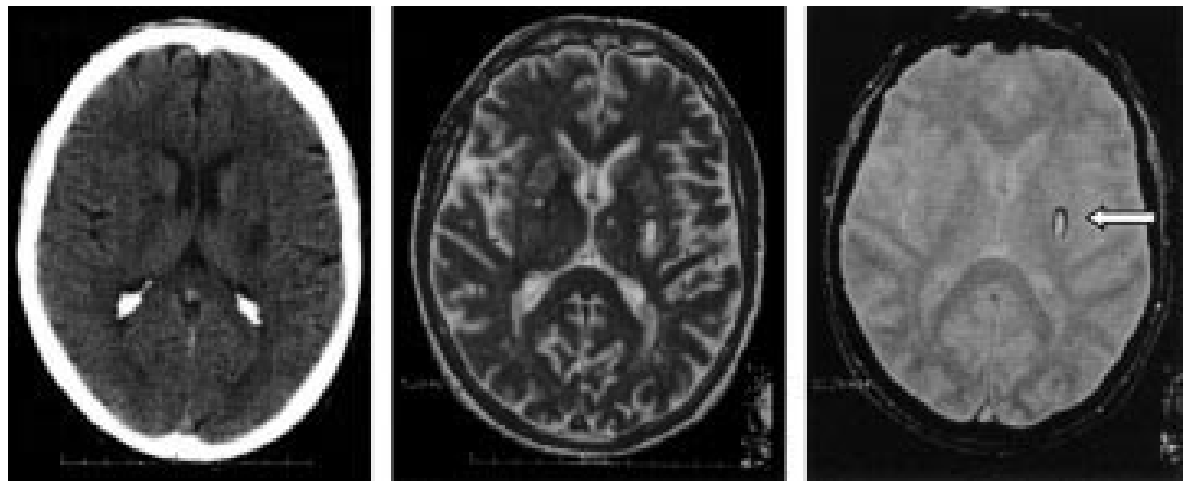

Figure 1 Brain images of a 60 year old man with right arm and leg weakness and facial numbness presenting at 21 days, when the only residual findings were reduced fine finger movements. Left panel: computed tomography (CT) of the brain: there is a low density area consistent with lacunar infarction in the left lentiform nucleus. Middle panel: T2 weighted image: there is a high intensity (white) lesion in the lentiform nucleus with a dark rim consistent with haemosiderin from a haemorrhage. Right panel: gradient echo magnetic resonance imaging: the dark rim around the hyperintensive lesion is more obvious (arrow) and confirms that the lesion was a primary intracerebral haemorrhage. 

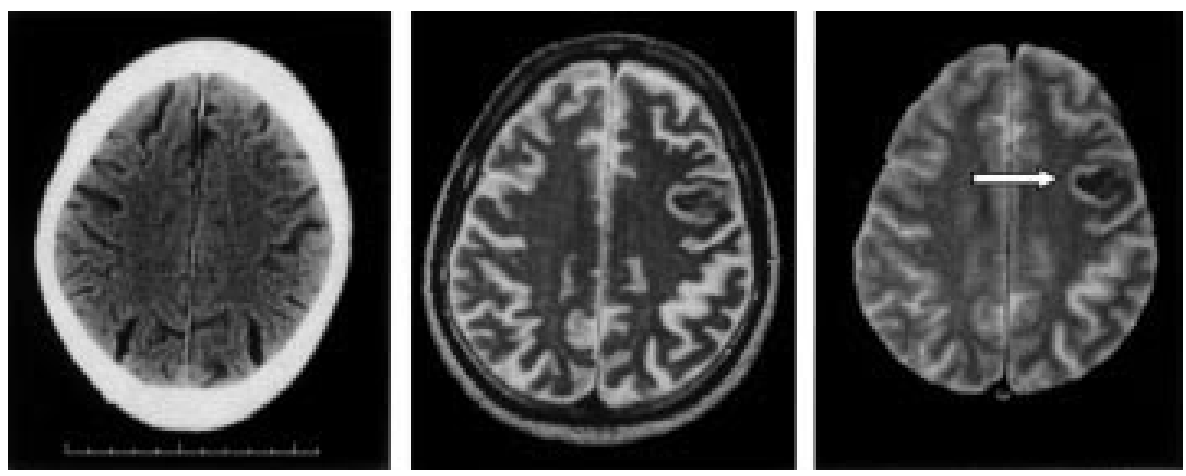

Figure 2 Brain images of a 70 year old man with expressive dysphasia that had almost completely resolved at presentation at 19 days. Left panel: computed tomography (CT) of the brain suggests no abnormality. Middle panel: T2 weighted image shows a hyperintense (white) gyrus with a central area of hypointensity (dark) area corresponding to the gyri and consistent with haemorrhagic transformation, the dark area being haemosiderin from petechial haemorrhage along a gyrus. Right panel: gradient echo magnetic resonance imaging: the hyperintense (dark) area is more obvious. The gyral pattern is consistent with intensive petechial haemorrhagic transformation of a parietal cortical infarct.

changes (fig 2). CT agreed in only two patients $(0.9 \% ; 0.1 \%$ to $3.1 \%)$. Corresponding findings on CT when haemorrhagic transformation was not identified were: recent haemorrhage (that is, the lesion was thought to be a primary intracerebral haemorrhage not haemorrhagic transformation) (1), recent infarct (10), old lesion probable infarct (1), and multiple periventricular lucencies and cortical atrophy only (1). In all but the last patient, the ischaemic lesion seen on CT was the same as that shown to be haemorrhagic on MRI. CT therefore missed the haemorrhagic changes seen on MRI in 12 of 15 cases ( $80 \%$ ).

The time from onset of symptoms to scan for both CT and MRI in patients found to have either recent haemorrhage or haemorrhagic transformation ranged from four to 56 days. The shortest time from stroke to scan when recent primary intracerebral haemorrhage was missed on CT was 11 days. The shortest time from stroke to scan when haemorrhagic transformation (albeit very minor) was missed on CT was four days. The two patients in whom CT correctly identified recent haemorrhage were scanned at nine and 14 days after the stroke (fig 3).

Signs suggestive of old asymptomatic haemorrhage not related in location to the current symptoms or signs were seen on 15 MRI scans $(6.6 \%)$ but on no CT scans. These were the only significant imaging findings in five patients $(2.3 \%)$. In the remaining 10 , these signs were seen alongside signs of recent infarction (8) and haemorrhagic transformation (2).

\section{Patient preference questionnaire}

Questionnaires were available for 192 patients (84\%); 82 (43\%) underwent CT first and 110 (57\%) underwent MRI first; 150 patients (78\%) preferred CT, $13(7 \%)$ preferred MRI, and
29 (15\%) expressed no preference. All patients felt they would agree to further CT if they had to, and $99 \%$ would agree to MRI, although four patients added that it would be under duress. Additional comments included: the friendliness and helpfulness of the staff (21), the MRI scanner was noisy (11), it was claustrophobic (6), the time taken for MRI was too long (5), and the MRI was frightening (2). One patient liked the noise of the MRI scanner, and one felt more secure in the MRI scanner and found it more relaxing than CT.

\section{DISCUSSION}

This is the largest ever direct comparison of CT with MRI in stroke. The frequency of primary intracerebral haemorrhage in our study population was $3.5 \%$ and of haemorrhagic transformation, $6.6 \%$. Thus acute haemorrhagic changes were found in about $10 \%$ of this population overall. The issue addressed in this study was not "does CT miss haemorrhages in patients presenting late after stroke?" but rather, "how many haemorrhages does CT miss and how late is late?". CT missed 17 of the 23 haemorrhages that could be related to the recent stroke $(74 \%)$ (and all 15 old haemorrhages identified on MRI). The proportion of patients with primary intracerebral haemorrhage in this study was low, reflecting the fact that this lesion generally causes more severe strokes, ${ }^{4}$ but the $95 \%$ confidence interval included the possibility of up to $6.8 \%$ of primary intracerebral haemorrhages. It is likely that the epidemiology studies to date have systematically underestimated the true proportion of strokes caused by primary intracerebral haemorrhage as in none was the scanning policy optimal. ${ }^{6}$ Thus the proportion of mild stroke, recurrent stroke,

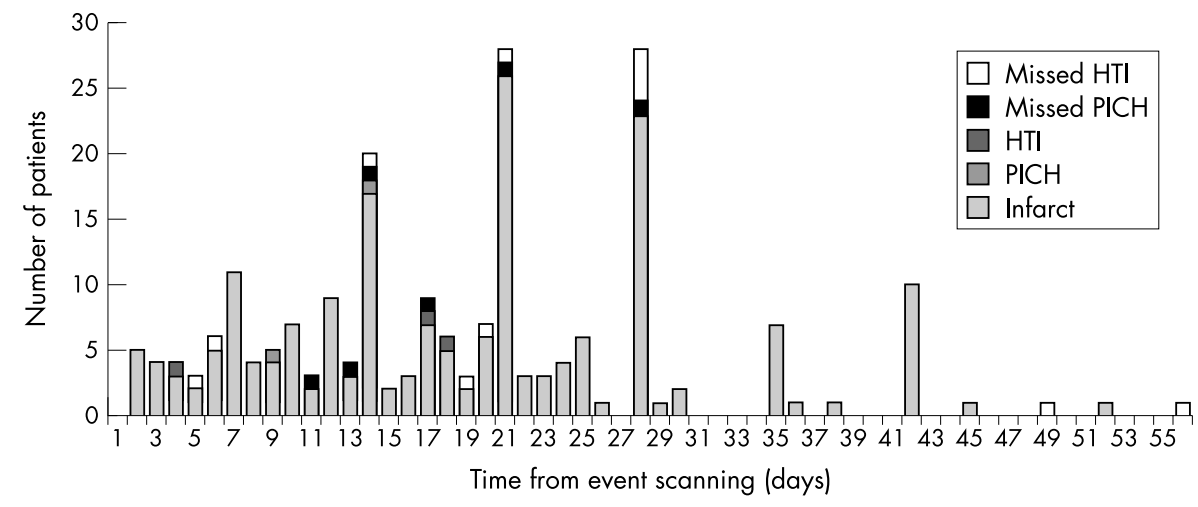

Figure 3 Patient scan findings in relation to time from onset of stroke symptoms to scanning (days). "Missed HTI" and "missed PICH" means that the haemorrhage was identified on magnetic resonance imaging but missed on computed tomography (CT); " $\mathrm{HTI}$ ", "PICH", and "infarct" represent lesions correctly identified on CT. HTI, haemorrhagic transformation of an infarct; PICH, primary intracerebral haemorrhage. 
or stroke in different age groups from this cause is unknown. However, the upper $95 \%$ confidence interval of our population $(6.8 \%)$ overlaps both the point estimates from the individual epidemiology studies (6\% to 24\%) and the lower 95\% confidence interval of the grouped epidemiology studies $(3 \%$ to $40 \%$ ), so it is within previous estimates.

\section{Public health impact}

Using MRI as the reference standard, the sensitivity of CT in detecting primary intracerebral haemorrhage and haemorrhagic transformation in this group of patients was $25 \%$ and $20 \%$, respectively. In absolute terms, CT misdiagnosed haemorrhage as infarction in 24/1000 patients with primary intracerebral haemorrhage (95\% CI, 14 to 37$)$. In the United Kingdom, around $15 \%$ of patients with stroke are not admitted to hospital-that is, roughly 22500 patients a year. ${ }^{8}$ Even among patients admitted to hospital, there may be delays of a week or more to CT scanning in the United Kingdom, as well as elsewhere, ${ }^{811}$ or CT may not be done at all. ${ }^{11}$ If only the patients with mild strokes were not scanned early enough using CT, then 540 patients with primary intracerebral haemorrhage (95\% CI, 315 to 833 ) and up to 2200 patients with any haemorrhage could be misdiagnosed and therefore managed inappropriately each year in the United Kingdom. Also, our data do not extend to a predominantly inpatient population where CT is not available early. In such a group, the frequency of missed primary intracerebral haemorrhage and haemorrhagic transformation is likely to be considerably higher, with even greater numbers misclassified as ischaemic stroke.

\section{Health care consequences}

Aspirin given to patients with ischaemic stroke reduces the risk of recurrent stroke or death by only about 9/1000 if given within the first 14 days of stroke. ${ }^{12}$ Among survivors of primary intracerebral haemorrhage, about $6.2 \%$ have a recurrent stroke, of which $75 \%$ are caused by further primary intracerebral haemorrhage, and $8.8 \%$ of these patients die each year. ${ }^{13}$ Patients taking aspirin for long term primary or secondary prevention who have a stroke are relatively more likely to have a primary intracerebral haemorrhage than patients who have a stroke while not taking aspirin. ${ }^{14}$ There are no direct data on long term administration of aspirin to patients with primary intracerebral haemorrhage. In two large acute stroke trials, some patients with primary intracerebral haemorrhage were inadvertently given a few doses of aspirin, which appeared to have little effect on the odds of further haemorrhage or death (recurrent haemorrhage, odds ratio 1.02 (95\% CI, 0.5 to 1.8 ); death, odds ratio 0.96 (0.62 to 1.5$)) .{ }^{15}$ These data, however, do not provide a reliable answer on the effect of long term antithrombotic treatment after primary intracerebral haemorrhage (these were very selected patients, they only received a few doses of aspirin in the acute phase, and the wide confidence intervals include the possibility of substantial harm). Despite the benefit of antithrombotic treatment in primary and secondary prevention of vascular ischaemic events, most physicians would probably wish to avoid aspirin, and certainly heparin or warfarin, in patients with known primary intracerebral haemorrhage (unless there was a very high risk of recurrent ischaemic events). Prevention would more probably be concentrated on other forms of risk reduction such as cholesterol or blood pressure lowering, ${ }^{16}$ cessation of smoking, and advice on diet and exercise. Furthermore, other forms of secondary prevention would be misguided. For example, these patients might undergo carotid endarterectomy (if they have a tight carotid stenosis) for secondary prevention. This would expose them to risk, with little chance of benefit, and be a further waste of health care resources.

\section{Implications for research}

There is not a single community based epidemiological study of stroke where CT scanning was done in all patients within eight days. ${ }^{6}{ }^{17} 18$ Rather, the median time to scanning was 18.5 days (95\% CI, 7 to 30 days) in $63 \%$ of the patients $(60 \%$ to $85 \%) .{ }^{6}$ Patients with mild strokes were one of the groups most likely not to undergo scanning at all. Any study of genetics or risk factors for haemorrhagic stroke will produce misleading results unless the imaging is adequate to classify the strokes accurately as ischaemic or haemorrhagic. Furthermore, previous asymptomatic haemorrhage was found on MRI in 15 patients $(6.6 \%)$, but in none using CT. Previous studies using MRI $^{19-23}$ (in 343 patients) found old haemorrhages in from $26 \%{ }^{22}$ to $68 \%^{21}$ of the patients. The implications of this for management of secondary prevention after stroke are unclear.

\section{Reasons for delays}

Our group of patients, which represents a fairly typical population presenting to a neurovascular clinic in a large city in the United Kingdom, was seen and scanned an average of three weeks after their stroke. The main reasons for the delay to presentation were delays in the patient presenting to their family practitioner, and between referral and being seen in the neurovascular clinic. This was despite clinics taking place twice a week and most patients being given an appointment within 10 days of referral. This cumulative delay between onset of symptoms and eventual scanning will have had a detrimental effect upon the sensitivity of CT for detecting primary intracerebral haemorrhage. ${ }^{24}{ }^{25}$ The earliest time after the event at which a primary intracerebral haemorrhage was missed on CT was 11 days. In order to have detected hyperdensity and so diagnosed primary intracerebral haemorrhage on CT, it would have been necessary to scan the patient several days earlier, but it is impossible to identify precisely the earliest time at which the primary intracerebral haemorrhage would have been missed. A reasonable estimate would be about eight days. The latest time primary intracerebral haemorrhage was identified on CT was 14 days. These findings suggest that CT should not be used more than about eight days after the onset of stroke symptoms if one wishes to exclude haemorrhage (as opposed to identifying a non-stroke lesion).

\section{Conclusions}

Our study highlights the importance of undertaking MRI if it is essential to identify primary intracerebral haemorrhage, and if the onset of symptoms was more than eight days previously. However, MRI remains far less accessible than CT, is more expensive, and is contraindicated in some patients. Waiting until MRI has been done before starting antithrombotic treatment in all patients presenting after eight days would create unacceptable delays in starting secondary prevention for many patients, reducing the benefit in the majority who actually have ischaemic stroke. It is therefore essential that patients should be encouraged to seek medical attention early after any stroke, and that family practitioners should refer rapidly to specialist clinics, which, along with imaging departments, should offer responsive and rapid access to medical and imaging assessment, if we are to achieve effective and cost-efficient secondary prevention of ischaemic (or haemorrhagic) stroke.

\section{ACKNOWLEDGEMENTS}

The work was funded by the Health Technology Assessment Panel of the UK National Health Service Research and Development Office. The views expressed in this paper are those of the authors and not of the funding agency.

\section{Authors' affiliations}

J M Wardlaw, S L Keir, M S Dennis, Department of Clinical

Neurosciences, Western General Hospital, Edinburgh, UK

Competing interests: none declared 


\section{REFERENCES}

1 Franke FL, Ramos LMP, van Gijn J. Development of multifocal haemorrhage in a cerebral infarct during computed tomography. $J$ Neurol Neurosurg Psychiatry 1990;53:531-2.

2 Dennis MS, Bamford JM, Molyneux AJ, et al. Rapid resolution of signs of primary intracerebral haemorrhage in computed tomograms of the brain. BM 1987;295:379-81.

3 Hung TP, Lee KY. Small intracerebral haemorrhage: a study of clinical manifestations and CT findings on 31 cases. Ann Acad Med Singapore $1985 ; 14: 22-31$

4 Poungvarin N, Viriyavejakul A, Komontri C. Siriraj stroke score and validation study to distinguish supratentorial intracerebral haemorrhage from infarction. BM 1991;302:1565-7.

5 Allen CM. Clinical diagnosis of the acute stroke syndrome. Q J Med 1983;52:515-23.

6 Keir S, Wardlaw JM, Warlow CP. Stroke epidemiology studies have underestimated the frequency of intracerebral haemorrhage. A systematic review of imaging in epidemiological studies. J Neurol 2002;249: 1226-31.

7 Wardlaw JM, Statham PF. How often is haemosiderin not visible on routine MRI following traumatic intracerebral haemorrhage? Neuroradiology 2000;42:81-4.

8 Ebrahim S, Redfern J. Stroke care - a matter of chance. A national survey of stroke services. London: The Stroke Association, 1999.

9 Bamford J, Sandercock P, Dennis M, et al. Classification and natural history of clinically identifiable subtypes of cerebral infarction. Lancet 1991;337:1521-6.

10 Cote R, Hachinski VC, Shurvell BL, et al. The Canadian neurological scale: a preliminary study in acute stroke. Stroke 1986;17:731-7.

11 Handshu R, Garling A, Heuschmann PU, et al. Acute stroke management in the local general hospital. Stroke $2001 ; 32: 866-70$.

12 Chen $\mathbf{Z}$, Sandercock P, Pan $\mathrm{H}$, et al. Indications for early aspirin use in acute ischaemic stroke. A combined analysis of 40000 randomised patients from the Chinese Acute Stroke Trial and the International Stroke Trial. Stroke 2000;31:1240-9.

13 Bailey RD, Hart RG, Benavente $O$, et al. Recurrent brain hemorrhage is more frequent than ischemic stroke after intracranial hemorrhage. Neurology 2001;56:773-7.
14 Jiang He, Whelton PK, Vu B, et al. Aspirin and risk of haemorrhagic stroke. A meta-analysis of randomised controlled trials. JAMA 1998;280:1930-5.

15 Keir S, Wardlaw J, Lewis S, et al, on behalf of CAST and IST collaborative groups. Antithrombotic therapy in patients with any form of intracranial haemorrhage: a systematic review of the available controlled studies. Cerebrovasc Dis 2002;14:197-206.

16 PROGRESS Collaborative Group. Randomised trial of a peridopril-based blood-pressure-lowering regimen among 6105 individuals with previous stroke or transient ischaemic attack. Lance 2001;358: 1033-41.

17 Thrift AG, Dewey HM, Macdonell RAL, et al. Incidence of the major stroke subtypes. Initial findings from the North East Melbourne Stroke Incidence Study (NEMESIS). Stroke 2001;32:1732-8.

18 Tanizaki Y, Kiyohara Y, Kato I, et al. Incidence and risk factors for subtypes of cerebral infarction in a general population. Stroke 2001:31:2616-22.

19 Offenbacher $\mathbf{H}$, Fazekas F, Schmidt R, et al. MR of cerebral abnormalities concomitant with primary intracerebral haematomas. Am J Neuroradiol 1996;17:573-8.

20 Tanaka A, Ueno Y, Nakayama Y, et al. Small chronic haemorrhages and ischaemic lesions in association with spontaneous intracerebral haematomas. Stroke 1999:30:1637-42.

21 Kinoshita T, Okudera T, Tamura H, et al. Assessment of lacunar hemorrhage associated with hypertensive stroke by echo-planar gradient-echo T2*-weighted MRI. Stroke 2000;31:1646-50.

22 Kwa VIH, Franke CL, Verbeeten B, et al. Silent intracerebral microhaemorrhages in patients with ischaemic stroke. Ann Neurol 1998:44:372-7

23 Greenberg SM, Finklestein SP, Schaefer PW. Petechial hemorrhages accompanying lobar hemorrhage: detection by gradient-echo MRI. Neurology 1996;46:1751-4.

24 Mayer TE, Schulte-Altedorneburg G, Droste DW, et al. Serial CT and MRI of ischaemic cerebral infarcts: frequency and clinical impact of haemorrhagic transformation. Neuroradiology 2000;42:233-9.

25 Steinbrich W, Gross-Fengels W, Krestin GP, et al. MRI examinations of intracranial haematomas. An analysis of its sensitivity evolution and source of bleeding. Fortschr Gebiete Rontgenstr Nuklearmed $1990 ; 152: 534-43$. 